\title{
Detection and management of depression in adult primary care patients in Hong Kong: a cross-sectional survey conducted by a primary care practice-based research network
}

Weng Yee Chin ${ }^{1 *}$, Kit TY Chan ${ }^{1}$, Cindy LK Lam¹, Samuel YS Wong ${ }^{2}$, Daniel YT Fong ${ }^{3}$, Yvonne YC Lo ${ }^{4}$, Tai Pong Lam ${ }^{1}$ and Billy CF Chiu ${ }^{5}$

\begin{abstract}
Background: This study aimed to examine the prevalence, risk factors, detection rates and management of primary care depression in Hong Kong.

Methods: A cross-sectional survey containing the PHQ-9 instrument was conducted on waiting room patients of 59 primary care doctors. Doctors blinded to the PHQ-9 scores reported whether they thought their patients had depression and their management.

Results: 10,179 patients completed the survey (response rate $81 \%$ ). The prevalence of PHQ-9 positive screening was 10.7\% (95\% Cl: 9.7\%-11.7\%). Using multivariate analysis, risk factors for being PHQ-9 positive included: being female; aged $\leq 34$ years; being unmarried; unemployed, a student or a homemaker; having a monthly household income < HKD\$30,000 (USD\$3,800); being a current smoker; having no regular exercise; consulted a doctor or Chinese medical practitioner within the last month; having $\geq$ two co-morbidities; having a family history of mental illness; and having a past history of depression or other mental illness. Overall, $23.1 \%$ of patients who screened PHQ-9 positive received a diagnosis of depression by the doctor. Predictors for receiving a diagnosis of depression included: having higher PHQ-9 scores; a past history of depression or other mental health problem; being female; aged $\geq 35$ years; being retired or a homemaker; being non-Chinese; having no regular exercise; consulted a doctor within the last month; having a family history of mental health problems; and consulted a doctor in private practice.

In patients diagnosed with depression, $43 \%$ were prescribed antidepressants, $11 \%$ were prescribed benzodiazepines, $42 \%$ were provided with counseling and $9 \%$ were referred, most commonly to a counselor.

Conclusion: About one in ten primary care patients screen positive for depression, of which doctors diagnose depression in approximately one in four. At greatest risk for depression are patients with a past history of depression, who are unemployed, or who have multiple illnesses. Patients most likely to receive a diagnosis of depression by a doctor are those with a past history of depression or who have severe symptoms of depression. Chinese patients are half as likely to be diagnosed with depression as non-Chinese patients. Over half of all patients diagnosed with depression are treated with medications.
\end{abstract}

Keywords: Mental health, Chinese, Depression, Epidemiology, Primary care, Prevalence, Screening, Detection, Practice-based research network

\footnotetext{
*Correspondence: chinwy@hku.hk

${ }^{1}$ Department of Family Medicine and Primary Care, The University of

Hong Kong, Hong Kong, China

Full list of author information is available at the end of the article
} 


\section{Background}

Depression is a common and serious disorder that impairs quality of life [1]. There are many barriers to recognizing and managing patients with depression, particularly in primary care where most patients with depressive disorders are seen [2-4]. Around the world, the prevalence of depressive disorders in primary care has been estimated to be between $10-20 \%$, of which around half remain undetected by doctors [5]. A recent household telephone survey conducted on a Hong Kong Chinese general population sample estimated the cross-sectional community prevalence for moderate to severe depression (defined as PHQ-9 score $>9$ ) as $4.3 \%$, however only $1.6 \%$ reported to have had a lifetime history of depression diagnosed by a doctor [6]. Similarly, in the 2003-2004 Hong Kong Population Health Survey, only $1.5 \%$ of adult respondents reported having a past diagnosis of depression by a doctor [7].

Primary care is the entry point for most people into the healthcare system and a common pathway to mental health care services. The choice of interventions offered by primary care physicians (PCPs) can have a significant impact both on patients' quality of life as well as on health service demands. Not all primary care patients with depression respond to antidepressants and current international guidelines on adult primary care depression recommend a 'stepped' approach which may involve watchful waiting, counselling, use of psychotropic medications, or referral for further psychological or psychiatric services. There is evidence that delivery of stepped care can improve patient outcomes [8-10].

Hong Kong is a Special Administrative Region of the People's Republic of China located in the Pearl Delta district, south of the Guangdong province of China. It has a population of approximately seven million of whom 95\% are of Chinese descent. As a result of its past as a British colony, Hong Kong is often referred to as a place where "East meets West". Despite this, the Chinese living in Hong Kong still hold many of the values, beliefs and behaviours typical of Chinese populations both from China and overseas which have their origins based on Taoism and Confucianism [11]. Hong Kong has a developed capitalist economy, with a gross domestic product of US\$301.6 billion of which about $5.5 \%$ is spent on healthcare and about $0.24 \%$ on mental health [12]. It has a two-tiered mixed medical economy and a pluralistic healthcare system. $80 \%$ of primary care is delivered through the 'fee-for-service' private sector with the remaining $20 \%$ delivered through governmentfunded General Out-Patient Clinics (GOPCs) which cater mainly for chronic disease management and for the elderly. In the private 'fee-for-service' setting, any patient can seek the care of any doctor for any illness, and there are no statutory post-graduate qualifications required for doctors to practice in primary care [13]. As little is currently known about the epidemiology, detection and management patterns for depression in Hong Kong's primary care setting, the objectives of this study are to:

1. Estimate the cross-sectional prevalence of depression in primary care and its associated risk factors;

2. Examine the detection rates for depression and the predictors for diagnosis of depression by a doctor;

3. Examine how primary care doctors manage patients with depression.

The findings of this study will be used to help guide mental health service planning and policy development, and will contribute to the overall knowledge on identification and management of depression in Chinese primary care patients.

\section{Methods}

\section{Study design}

This was a cross-sectional observational study using a primary care practice-based research network. This study formed the baseline for a longitudinal cohort study examining the outcomes of depression in primary care [14].

\section{Participants and sampling}

Primary care physicians (PCPs) working in clinics across Hong Kong were invited to join our research network. Doctors were recruited using the mailing list of the Hong Kong College of Family Physicians (HKCFP) and consisted of PCPs working in solo and group private practice, government-funded General Out-Patient Clinics of the Hospital Authority of Hong Kong, and non-profit, non-governmental organizations (NGO).

All eligible patients presenting to the study doctor on one randomly selected day each month over a 12-month recruitment period were invited to participate. The recruitment days were randomly generated but excluded Sundays and public holidays. A twelve-month baseline collection period was used to account for seasonal variability and to improve representation of the primary care case load. All consecutive waiting room patients were approached to join the study. Patients were excluded if they were $<18$ years, did not speak or understand English, Cantonese or Mandarin, had cognitive or communication difficulties, had already been recruited to the study, or did not consult the study doctor.

A coded, anonymous questionnaire containing items on age, gender, marital status, ethnicity, education, household income, district of residence, co-existing medical conditions, family history of mental illness, previous doctordiagnosed depression or other mental illness, recent mental health or other health care service use, and the PHQ-9 was 
self-completed by participants in the PCP's waiting room. Questionnaires were available in English and Chinese. A bilingual research assistant was present to explain the study, obtain consent, and distribute and collect questionnaires. If subjects had difficulty completing the questionnaire, the research assistant helped to administer the questionnaire. Doctors who were blinded to the questionnaire results were asked to complete a clinical data collection form for each patient recruited into the study.

\section{Study instruments}

\section{A. The Patient Health Questionnaire 9 (PHQ-9).}

The PHQ-9 is a self-reported depression component of the Primary Care Evaluation of Mental Disorder Procedure (PRIME-MD) [15] which has been validated for use in primary care for diagnosis of depression. It scores each of the 9 DSM-IV criteria from 0 (not at all) to 3 (nearly every day). It has been calibrated against the Chinese Hamilton Depression Scale for screening use in Hong Kong's primary care setting. Using a cut-off score of 9, the PHQ-9 has a sensitivity of $80 \%$ and specificity of 92\% for the diagnosis of depression [16]. The PHQ-9 can also be used to evaluate the severity of symptoms (score 1-4 minimal, 5-9 mild, 10-14 moderate, 15-19 moderately severe, 20-27 severe) and has been used for monitoring symptom progression or remission over time [17].

B. Personal data questions on socio-demography and co-morbidity were adapted from previous primary care patient surveys performed in Hong Kong [18].

C. Doctor's data collection form. Doctors were asked to record the patient's presenting problem, their opinion on whether they felt the patient had depression operationally defined as a "clinically significant depressive disorder", and whether the diagnosis was new or old. If the doctor reported a diagnosis of depression, the doctor was asked to provide further information regarding the duration of illness, and their management.

\section{Sample size calculation}

Details of the sample size estimation required to calculate the outcome measures of the cross-sectional and longitudinal cohort study have already been described in our published study protocol [14]. For the estimation of the prevalence of depression by PHQ-9, the sample size was estimated to ensure an error of $<2 \%$ for an anticipated prevalence of $20 \%$. Without the consideration of the design effect due to cluster sampling by practice, 1540 subjects were needed in total with a 95\% confidence interval. The intraclass correlation (ICC) for the intracluster correlation was taken as 0.02 which was more conservative than the one reported in the Diamond study [19]. With 50 practices and after accounting the design effect due to cluster sampling, 80 subjects per practice were needed.

\section{Analysis}

The prevalence of depression was estimated from the patient's screening PHQ-9 scores (using a cut-off score $>9$ to define a positive case) with a $95 \%$ confidence interval taking into account the clustering by practice effect [12]. Chi-square and multiple logistic regression analyses were conducted to identify the patient risk factors associated with PHQ-9 positive screening and the patient predictors for being diagnosed with depression by the doctor. As demographic risk factor identification was the main goal of analysis, all demographic factors were kept in the regression model, whilst other patient clinical characteristics (such as PHQ-9 severity, patient-reported past history of depression and patient-reported past history of other mental illness) were entered in the regression model as a second block to allow for separate comparison. These patient characteristics were examined in separate blocks as they were considered to be theoretically highly linked to the dependent variables, and their inclusion together may result in a reduction of the significance of the other demographic factors which we wished to assess. Multicollinearity diagnostics were performed for all regression models [20]. Only patients with full data were used in the model. Imputation or other substitution methods were not used as there was a sufficiently large sample size to perform all analyses. Management of depression was descriptively analyzed based on the doctor's reported management of patients who they diagnosed with depression. All analyses were performed using SPSS 21.

\section{Ethics approvals}

This study received approvals by the Institutional Review Board of the University of Hong Kong/Hospital Authority Hong Kong West Cluster, the Research Committee of Evangel Hospital, the Research Committee of Hong Kong Sanatorium and Hospital, the Research Committee of Matilda International Hospital, the Research Ethics Committee for Hong Kong Hospital Authority Kowloon East Cluster and Kowloon Central Cluster, and the Joint Chinese University of Hong Kong and Hong Kong Hospital Authority New Territories East Cluster Clinical Ethics Review Committee.

\section{Results}

\section{Study subjects}

Fifty-nine PCPs participated in this study. The doctors' clinics were located across all three geographical regions of Hong Kong (Hong Kong Island, Kowloon and New Territories). Forty four of the study doctors had Fellowship 
qualifications in General Practice (or equivalent); one had a Fellowship qualification in Surgery; one had a Fellowship qualification in Emergency Medicine; whilst the remaining doctors had no postgraduate specialty qualifications. Fourteen of the study doctors had completed a Post-Graduate Diploma in Psychological Medicine.

Recruitment of patients occurred between October 2010 and January 2012. A total of 10,179 patients consented to participate with a response rate of $81 \%$ of all eligible subjects approached. Public sector Government Out-patient Clinic (GOPC) patients made up $26 \%$ of the subjects, whilst the remainder were recruited from private practices and Non-Governmental Organizations (NGOs) aligning proportionately with the overall delivery of primary care in Hong Kong [21]. Amongst all subjects, the mean age was 49 years; $95.7 \%$ were ethnically Chinese; and $56.6 \%$ were female. Demographic information on the doctors, clinics and subjects are shown in Table 1.

\section{Prevalence of depressive symptoms}

Using a PHQ-9 cut-off score $>9$ to define a positive case and taking into account the effect of clustering by doctor, the prevalence for PHQ-9 positive screened depression was estimated at $10.7 \%$ (95\% CI: 9.7\%-11.7\%). The prevalence was higher in patients recruited from public sector settings $(12.0 \%)$ than private $(10.1 \%)$; in women $(12.2 \%)$ than men (8.1\%); and in patients under the age of 35 years (12.3\%) than those who were 35 years or over $(9.8 \%)$. Mean PHQ-9 scores were higher in women than men in all age groups. Mean PHQ-9 scores became lower with increasing age in both sexes in a step-wise fashion as shown in Table 2.

\section{Patient factors associated with $\mathrm{PHQ}-9$ positive screening}

Patient factors associated with PHQ-9 positive screening, the corresponding chi-square statistics, adjusted odds ratios (OR) derived from multiple logistic regression analyses, and model evaluation statistics are shown in Table 3. Risk factors for PHQ-9 positive screening included being female; being aged 18-34 (relative to aged 55 above); being unmarried; being unemployed, a student or a homemaker (relative to being employed); having a household income under HKD\$30,000 per month; being a current smoker; having no exercise habit; having consulted a doctor or Chinese medical practitioner in the preceding four weeks; having $\geq$ two co-morbidities; and having a family history of mental health problems. At highest risk for being PHQ-9 positive were patients who were unemployed (2.74-fold greater increased risk than employed patients), and patients with $\geq$ two comorbidities (2.45-fold increased risk than patients with no co-existing illness). All patient demographic factors which were significant in the chi-square analysis remained
Table 1 Demographic information on doctors and patient

\begin{tabular}{|c|c|}
\hline Doctors and clinic: $(\mathrm{N}=59)$ & $\begin{array}{l}\text { Patient demographic: } \\
(\mathrm{N}=10.179)\end{array}$ \\
\hline Gender & Gender \\
\hline Male 42 (712\%) & Male $40.7 \%$ \\
\hline Female 17 (28.8\%) & Female $56.6 \%$ \\
\hline Age & Age \\
\hline \multirow[t]{2}{*}{$\begin{array}{l}\text { Mean age } 44.9 \text { years } \\
\text { (range } 30-75 \text { years) }\end{array}$} & $\begin{array}{l}\text { Mean age } 49.0 \text { years } \\
\text { (range 18-103 years) }\end{array}$ \\
\hline & $18-24$ years $6.8 \%$ \\
\hline Place of graduation & $25-24$ years $18.4 \%$ \\
\hline Hong Kong 46 (78.0\%) & $35-44$ years $16.9 \%$ \\
\hline Overseas 13 (22.0\%) & $45-54$ years $17.5 \%$ \\
\hline \multirow{3}{*}{$\begin{array}{l}\text { (China 2; UK 3; Australia/ } \\
\text { NZ 7; other 1) }\end{array}$} & $55-64$ years $15.7 \%$ \\
\hline & $65+$ years $20.3 \%$ \\
\hline & Ethnicity \\
\hline Post-graduate qualifications & Chinese $95.7 \%$ \\
\hline Fellowship in family medicine & Non-Chinese 3.9\% \\
\hline
\end{tabular}

Household monthly income (HKD)

Diploma in psychological
medicine $14(23.7 \%)$
ocation of clinic*
Hong Kong Island 26 (44.1\%)
Kowloon 18 (30.5\%)
New Territories 15 (25.4\%)

Territories 15 (25.4\%)

Type of practice
Private solo 21 (35.6\%)
Private group $12(20.3 \%)$
Private hospital $11(18.6 \%)$
Government clinic 11 (18.6\%)
NGO $3(5.1 \%)$
University $1^{\dagger}(1.7 \%)$

$\leq \$ 500012.2 \%$

$\$ 5,001-\$ 10,0007.5 \%$

$>\$ 40,00021.2 \%$

Marital status

Single $26.6 \%$

Married59.8\%

Widowed $7.6 \%$

\section{Education level}

Primary $16.0 \%$

Secondary $40.5 \%$
$\$ 10,001-\$ 20,00017.8 \%$

$\$ 20,001-\$ 30,00015.1 \%$

$\$ 30,001-\$ 40,00010.5 \%$

Separated/divorced 3.2\%

No formal schooling $7.7 \%$

Tertiary $32.7 \%$

District of Residence*

Hong Kong Island $40.1 \%$

Kowloon 22.0\%

New Territories 34.1\%

Mainland China $0.3 \%$

*Hong Kong is divided into three major geographical regions: Hong Kong Island, Kowloon and the New Territories.

${ }^{\dagger}$ One study doctor changed practice type from private solo to university health services during the study period. 
Table 2 PHQ-9 severity and mean scores among study participants $(\mathrm{N}=10,179)$

\begin{tabular}{lccc}
\hline & Overall & Male & Female \\
\hline PHQ-9 scores by severity (score range) & & & \\
Minimal (0-4) & $61.1 \%$ & $65.4 \%$ & $59.0 \%$ \\
Mild (5-9) & $25.3 \%$ & $24.5 \%$ & $26.0 \%$ \\
Moderate (10-14) & $7.5 \%$ & $6.0 \%$ & $8.3 \%$ \\
Moderately severe (15-19) & $2.3 \%$ & $1.6 \%$ & $2.8 \%$ \\
Severe (20-27) & $0.8 \%$ & $0.6 \%$ & $1.0 \%$ \\
Mean PHQ-9 scores by age and sex & & & 5.42 \\
18-24 yrs & 5.33 & 5.20 & 5.23 \\
25-34 yrs & 5.09 & 4.87 & 4.61 \\
35-44 yrs & 4.44 & 4.20 & 4.55 \\
45-54 yrs & 4.09 & 3.49 & 4.09 \\
55-64 yrs & 3.68 & 3.14 & 4.09 \\
65+ yrs & 3.57 & 3.01 & 3.98 \\
\hline
\end{tabular}

Note. PHQ-9 scale score ranges from 0 to 27 with higher score indicating more frequent occurrence of depressive symptoms. A person scoring 10 or above is classified as 'PHQ-9screened positive'.

Total count can be less than $100 \%$ due to missing information provided.

statistically significant in the multiple logistic regression analysis except for service sector.

With all patient demographic variables retained in block 1 , two more patient characteristics were then included in the second block to assess for additional significance: 'patient-reported previous diagnosis of depression' and 'patient-reported previous diagnosis of other mental illnesses'. The presence of a previous diagnosis of depression was associated with a 4.1 -fold increased risk of being PHQ-9 positive, whilst the presence of a previous diagnosis of other mental illness was associated with a 1.5-fold increased risk. All patient demographic factors that were significant in the previous analysis remained significant with the inclusion of block 2 variables. Multicollinearity was not evident among the factors analyzed as demonstrated by all variance inflation factors being $<4$ and no condition indices $>30$. The predictors as a set explained $14.3 \%$ of model variance (Nagelkerke R-square $=0.143$ ); the model chi-square testing was significant and Hosmer \& Lemeshow testing (HL test) was not significant indicating an acceptable fit between the predicted and actual outcomes.

\section{Detection of depression by doctors}

The study doctors, blinded to their patients' PHQ-9 screening scores, were asked to report whether they thought their patients had depression. Among all patients with PHQ-9 score $>9(n=1076), 23.1 \%$ received a diagnosis of depression by the doctor. Detection rates for depression increased with higher PHQ-9 scores (Figure 1). Among patients with a moderately severe levels of depression (PHQ-9 score $\geq 15$ ), 30.8\% were diagnosed as having depression. Among patients with severe levels of depression (PHQ-9 score $\geq 20$ ), $49.4 \%$ were diagnosed as having depression. In patients with a doctors diagnosis of depression and had a fully completed PHQ-9 score $(n=602)$, $58.6 \%$ screened negative for depression (PHQ-9 score $\leq 9$ ).

\section{Predictors for diagnosis of depression by a doctor}

Multiple logistic regression analysis was conducted to identify the predictors for doctor diagnosis of depression. As before, the significance of basic demographic variables and additional clinical characteristics were examined in different blocks. The distributions of each patient variable and multivariate statistics are shown in Table 4. Patient demographic factors significantly associated with higher likelihood of being diagnosed with depression included: being female, being aged 35 years and above; being unemployed, retired, or home-maker (relative to being employed); being non-Chinese, having no regular exercise pattern; having seen a doctor within the last 4 weeks; and having two or more co-morbidities. Patients most likely to receive a diagnosis of depression were unemployed (3.53-fold greater likelihood), or non-Chinese (2.52-fold greater likelihood).

With all demographic variables retained in block 1 , three additional patient variables were analyzed in the second block: PHQ-9 severity classification (minimal, mild, moderate, moderately severe and severe), patient-reported previous diagnosis of depression and patient-reported previous diagnosis of other mental health problem. Presence of moderately severe depression (PHQ-9 score of 20-27) was associated with an additional 8.53-fold increased likelihood of receiving a diagnosis of depression and the presence of severe depression (PHQ-9 score $\geq 20$ ) was associated with a 10.42-fold increase. Having a patientreported past diagnosis of depression was associated with a 10.16-fold increased likelihood of receiving diagnosis of depression, and having a patient-reported past history of other mental illness was associated with a 2.89-fold increase. Most patient demographic factors remained statistically significant after the inclusion of the block 2 variables with the exceptions of being unemployed and having $\geq$ two co-morbidities which changed from significant to insignificant, and service sector which changed from insignificant to significant. Multicollinearity among the factors analyzed was not evident. The whole set of predictors explained $32.0 \%$ of the model variance as suggested by Nagelkerke R-square value. A better model fit was supported by the significant model chi-square test, while the HL test became significant after the inclusion of second block variables.

\section{Management of depression}

In patients with diagnosed depression, doctors were asked to record their management using a tick-box selection 
Table 3 Associations between patient factors and PHQ-9 screening outcome

\begin{tabular}{|c|c|c|c|c|c|}
\hline \multirow{3}{*}{$\begin{array}{l}\text { Patient factor } \\
\text { Gender }\end{array}$} & \multirow{3}{*}{$\begin{array}{l}\text { PHQ + ve, } \\
\text { n (\%) }\end{array}$} & \multirow{3}{*}{$\begin{array}{l}\text { PHQ-ve, } \\
\text { n (\%) }\end{array}$} & \multirow{3}{*}{$\begin{array}{l}X^{2}(\mathbf{d f}) \\
44.41(1)^{* *}\end{array}$} & \multirow{2}{*}{\multicolumn{2}{|c|}{ 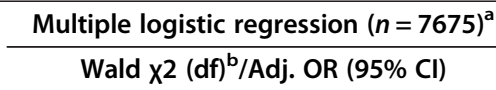 }} \\
\hline & & & & & \\
\hline & & & & $11.24(1)^{* *}$ & $4.55(1)^{*}$ \\
\hline Male & $336(32.4)$ & $3721(43.2)$ & & 1.00 & 1.00 \\
\hline Female & $702(67.6)$ & $4897(56.8)$ & & $1.36(1.14-1.62)$ & $1.22(1.02-1.46)$ \\
\hline Age group & & & $17.11(2)^{* *}$ & $9.99(2)^{* *}$ & $11.89(2)^{* *}$ \\
\hline $18-34$ yrs & $316(31.1)$ & $2201(26.0)$ & & 1.00 & 1.00 \\
\hline $35-54$ yrs & $371(36.5)$ & $3045(35.9)$ & & $0.95(0.76-1.18)$ & $0.87(0.70-1.09)$ \\
\hline $55+y r s$ & $329(32.4)$ & $3232(38.1)$ & & $0.63(0.46-0.87)$ & $0.58(0.42-0.80)$ \\
\hline Education & & & $0.45(1)$ & $0.01(1)$ & $0.09(1)$ \\
\hline Secondary or above & $772(75.0)$ & $6514(75.9)$ & & 1.00 & 1.00 \\
\hline Primary or no formal education & $258(25.0)$ & $2069(24.1)$ & & $0.99(0.78-1.25)$ & $1.04(0.82-1.32)$ \\
\hline Marital status & & & $71.62(1)^{* *}$ & $28.18(1)^{* *}$ & $24.14(1)^{* *}$ \\
\hline Married & $508(49.3)$ & $5394(62.9)$ & & 1.00 & 1.00 \\
\hline Single/separated/divorced/widowed & $523(50.7)$ & $3188(37.1)$ & & $1.62(1.35-1.93)$ & $1.57(1.31-1.88)$ \\
\hline Working Status & & & $83.98(4)^{* *}$ & $32.09(4)^{* *}$ & $22.70(4)^{* *}$ \\
\hline Employed & $582(57.6)$ & $5395(63.5)$ & & 1.00 & 1.00 \\
\hline Unemployed & $48(4.7)$ & $116(1.4)$ & & $2.74(1.79-4.18)$ & $2.31(1.48-3.60)$ \\
\hline Retired & $201(19.8)$ & $1833(21.6)$ & & $0.97(0.72-1.31)$ & $0.95(0.70-1.29)$ \\
\hline House-maker & $129(12.7)$ & $899(10.6)$ & & $1.33(1.01-1.75)$ & $1.30(0.98-1.73)$ \\
\hline Student & $52(5.1)$ & $248(2.9)$ & & $1.70(1.17-2.47)$ & $1.62(1.11-2.37)$ \\
\hline Household monthly income & & & $75.00(1)^{* *}$ & $28.93(1)^{* *}$ & $27.23(1)^{* *}$ \\
\hline More than $\mathrm{HK} \$ 30,000$ & $220(24.6)$ & $2951(39.4)$ & & 1.00 & 1.00 \\
\hline HK30,000 or below & $676(75.4)$ & $4539(60.6)$ & & $1.66(1.38-2.00)$ & $1.65(1.37-2.00)$ \\
\hline Ethnicity & & & $0.01(1)$ & $1.08(1)$ & $0.16(1)$ \\
\hline Chinese & $1039(96.3)$ & $8471(96.3)$ & & 1.00 & 1.00 \\
\hline Non-Chinese & $40(3.7)$ & $321(3.7)$ & & $1.27(0.81-1.98)$ & $1.10(0.69-1.74)$ \\
\hline Smoking habit & & & $19.24(1)^{* *}$ & $10.09(1)^{* *}$ & $8.90(1)^{* *}$ \\
\hline Non- or ex-smoker & $847(82.6)$ & $7511(87.4)$ & & 1.00 & 1.00 \\
\hline Current smoker & $179(17.4)$ & 1079 (12.6) & & $1.44(1.15-1.81)$ & $1.42(1.13-1.79)$ \\
\hline Alcohol use & & & $0.18(1)$ & $0.40(1)$ & $0.26(1)$ \\
\hline Less than once a week or never & $904(87.9)$ & $7510(87.4)$ & & 1.00 & 1.00 \\
\hline More than once a week & $125(12.1)$ & $1084(12.6)$ & & $1.08(0.85-1.39)$ & $1.07(0.83-1.38)$ \\
\hline Regular exercise pattern & & & $57.12(1)^{* *}$ & $29.46(1)^{* *}$ & \\
\hline Yes & $647(63.2)$ & $6361(74.3)$ & & 1.00 & 1.00 \\
\hline No exercise at all & $377(36.8)$ & $2204(25.7)$ & & $1.58(1.34-1.86)$ & $1.58(1.33-1.86)$ \\
\hline Seen a doctor in past 4 weeks & & & $94.67(1)^{* *}$ & $41.12(1)^{* *}$ & $30.88(1)^{* *}$ \\
\hline No & $356(33.0)$ & $4276(48.7)$ & & 1.00 & 1.00 \\
\hline More than once & $723(67.0)$ & $4512(51.3)$ & & $1.70(1.45-2.00)$ & $1.60(1.36-1.89)$ \\
\hline Seen a TCM practitioner in past 4 weeks & & & $87.93(1)^{* *}$ & $32.20(1)^{* *}$ & $31.28(1)^{* *}$ \\
\hline No & $751(69.6)$ & $7176(81.6)$ & & 1.00 & 1.00 \\
\hline More than once & $328(30.4)$ & $1615(18.4)$ & & $1.70(1.41-2.03)$ & $1.70(1.41-2.05)$ \\
\hline
\end{tabular}


Table 3 Associations between patient factors and PHQ-9 screening outcome (Continued)

\begin{tabular}{|c|c|c|c|c|c|}
\hline Disease co-morbidity & & & $71.11(2)^{* *}$ & $63.57(2)^{* *}$ & $48.96(2)^{* *}$ \\
\hline None & $437(42.3)$ & $4319(50.2)$ & & 1.00 & 1.00 \\
\hline One & $223(21.6)$ & $2207(25.7)$ & & $1.25(1.01-1.55)$ & $1.19(0.96-1.48)$ \\
\hline Two or more & $374(36.2)$ & $2071(24.1)$ & & $2.45(1.95-3.08)$ & $2.22(1.76-2.80)$ \\
\hline Family history mental illness & & & $37.88(1)^{* *}$ & $31.05(1)^{* *}$ & $16.62(1)^{* *}$ \\
\hline No & $869(85.8)$ & 7716 (91.6) & & 1.00 & 1.00 \\
\hline Yes & $144(14.2)$ & $704(8.4)$ & & $1.89(1.51-2.36)$ & $1.62(1.28-2.04)$ \\
\hline Service sector & & & $8.37(1)^{* *}$ & $2.49(1)$ & \\
\hline Public & $318(29.5)$ & $2232(25.4)$ & & 1.00 & 1.00 \\
\hline Private & $761(70.5)$ & $6560(74.6)$ & & $0.85(0.70-1.04)$ & $0.85(0.70-1.05)$ \\
\hline Self-reported depression history ${ }^{c}$ & & & $420.70(1)^{* *}$ & & $125.97(1)^{* *}$ \\
\hline No & $795(78.0)$ & $8104(95.1)$ & & & 1.00 \\
\hline Yes & $224(22.0)$ & $420(4.9)$ & & & $4.10(3.20-5.24)$ \\
\hline Self-reported other mental illness history ${ }^{c}$ & & & $110.70(1)^{* *}$ & & $4.82(1)^{*}$ \\
\hline No & $888(91.1)$ & $8195(97.4)$ & & & 1.00 \\
\hline Yes & $87(8.9)$ & $219(2.6)$ & & & $1.51(1.05-2.17)$ \\
\hline
\end{tabular}

${ }^{*} p<.05 ;{ }^{* *} p<.01$.

${ }^{a}$ Model Chi-square $=546.73, \mathrm{df}=22, p<.001 ;$ Nagelkerke R-square $=0.143 ;$ Hosmer \& Lemeshow Test, Chi-square $=14.42, \mathrm{df}=8, p=0.072$.

${ }^{b}$ Wald test statistics are shown in italic.

${ }^{\mathrm{C}}$ Factors entered in second block of the regression model; Block Chi-square $=141.46, \mathrm{df}=2, \mathrm{p}<.001$.

including medications, counselling, referral, observation and planned follow-up. Management was reported for 618 subjects. Overall, $50.6 \%$ of patients were prescribed medications (of which $84.0 \%$ were prescribed antidepressants) and $41.9 \% \%$ provided counselling (of which $83 \%$ were provided with supportive therapy). Another health care provider was involved in $39.3 \%$ of patients: new referrals were provided in $8.6 \%$, whilst $30.7 \%$ were already receiving care from another professional. The most common referral destinations were to a counselor (34.0\%) or to a government-funded Hospital Authority specialist psychiatric service (22.6\%). Only four patients (7.5\% of those referred) were to a private psychiatrist.

Management patterns were slightly different in patients with and without a self- reported history of previous diagnosis of depression. Patients who reported a depression history were more likely having received care from another professional (46.0\% vs. $17.8 \%)$, more likely be prescribed medication by the study doctor (59.1\% vs. $41.6 \%)$, and less likely be observed (15.0\% vs. $39.6 \%)$. Their PHQ-9 scores were significantly higher than those who reported no depression history $(t=2.19, d f=527, p=0.029)$.

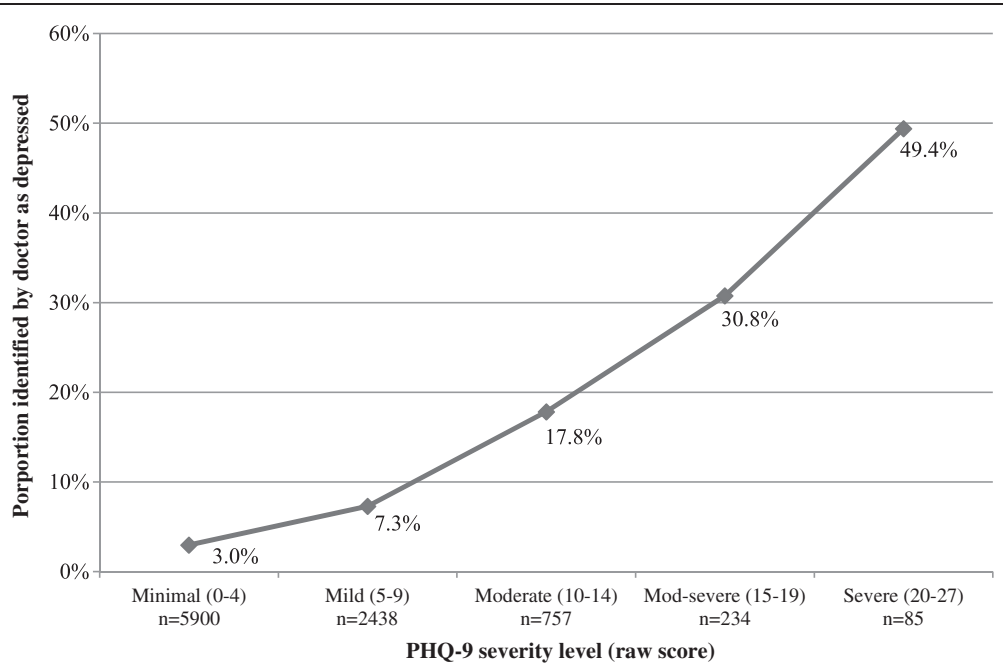

Figure 1 Detection of depression stratified by PHQ-9 sererity level. 
Table 4 Associations between patient factors and doctor detection

\begin{tabular}{|c|c|c|c|c|c|}
\hline \multirow{3}{*}{$\begin{array}{l}\text { Patient factor } \\
\text { Gender }\end{array}$} & \multirow{3}{*}{$\begin{array}{l}\text { Detected, } \\
\text { n (\%) }\end{array}$} & \multirow{3}{*}{$\begin{array}{l}\text { Not detected, } \\
\mathrm{n}(\%)\end{array}$} & \multirow{3}{*}{$\begin{array}{l}2 \text { (df) } \\
70.80(1)^{* *}\end{array}$} & \multirow{2}{*}{\multicolumn{2}{|c|}{$\begin{array}{c}\text { Multiple logistic regression }(n=7346)^{\mathrm{a}} \\
\text { Wald } X^{2}(d f)^{\mathrm{b}} / \text { Adj. OR }(95 \% \mathrm{Cl})\end{array}$}} \\
\hline & & & & & \\
\hline & & & & $23.70(1)^{* *}$ & $4.38(1)^{*}$ \\
\hline Male & $155(25.5)$ & $3795(42.9)$ & & 1.00 & 1.00 \\
\hline Female & $453(74.5)$ & $5053(57.1)$ & & $1.89(1.46-2.44)$ & $1.35(1.02-1.78)$ \\
\hline Age group & & & $34.76(2)^{* *}$ & $10.08(2)^{* *}$ & $7.94(2)^{*}$ \\
\hline $18-34$ yrs & $102(17.1)$ & $2351(27.0)$ & & 1.00 & 1.00 \\
\hline $35-54$ yrs & $216(36.2)$ & $3120(35.9)$ & & $1.71(1.23-2.39)$ & $1.65(1.14-2.39)$ \\
\hline $55+y r s$ & $279(46.7)$ & $3225(37.1)$ & & $1.61(1.05-2.49)$ & $1.86(1.14-3.01)$ \\
\hline Education & & & $23.68(1)^{* *}$ & $0.17(1)$ & $0.03(1)$ \\
\hline Secondary or above & $407(67.5)$ & $6721(76.3)$ & & 1.00 & 1.00 \\
\hline Primary or no formal education & $196(32.5)$ & $2090(23.7)$ & & $0.94(0.71-1.25)$ & $1.03(0.75-1.41)$ \\
\hline Marital status & & & $1.73(1)$ & $0.80(1)$ & $0.40(1)$ \\
\hline Married & $355(59.1)$ & $5422(61.5)$ & & 1.00 & 1.00 \\
\hline Single/Separated/Divorced/Widowed & $246(40.9)$ & $3391(38.5)$ & & $1.11(0.88-1.41)$ & $0.92(0.70-1.20)$ \\
\hline Working Status & & & $117.95(4)^{* *}$ & $38.85(4)^{* *}$ & $20.62(4)^{* *}$ \\
\hline Employed & $260(44.4)$ & $5578(64.0)$ & & 1.00 & 1.00 \\
\hline Unemployed & $26(4.4)$ & $135(1.5)$ & & $3.53(2.06-6.05)$ & $1.56(0.78-3.13)$ \\
\hline Retired & $167(28.5)$ & $1827(21.0)$ & & $2.00(1.40-2.84)$ & $2.16(1.45-3.21)$ \\
\hline House-maker & $115(19.6)$ & $902(10.3)$ & & $2.02(1.46-2.80)$ & $2.05(1.41-2.97)$ \\
\hline Student & $18(3.1)$ & $275(3.2)$ & & $1.62(0.83-3.17)$ & $1.18(0.56-2.46)$ \\
\hline Household monthly income & & & $19.86(1)^{* *}$ & $3.56(1)$ & $0.07(1)$ \\
\hline More than $\mathrm{HK} \$ 30,000$ & $146(28.5)$ & 2947 (38.3) & & 1.00 & 1.00 \\
\hline HK30,000 or below & $367(71.5)$ & $4746(61.7)$ & & $1.27(0.99-1.63)$ & $1.04(0.79-1.37)$ \\
\hline Ethnicity & & & $1.82(1)$ & $12.53(1)^{* *}$ & \\
\hline Chinese & $603(95.3)$ & $8703(96.3)$ & & 1.00 & 1.00 \\
\hline Non-Chinese & $30(4.7)$ & $333(3.7)$ & & $2.52(1.51-4.20)$ & $2.01(1.11-3.63)$ \\
\hline Smoking habit & & & $1.16(1)$ & $0.86(1)$ & $0.10(1)$ \\
\hline Non- or ex-smoker & $537(88.5)$ & $7656(87.0)$ & & 1.00 & 1.00 \\
\hline Current smoker & $70(11.5)$ & $1149(13.0)$ & & $1.18(0.83-1.67)$ & $0.94(0.64-1.39)$ \\
\hline Alcohol use & & & $4.99(1)^{*}$ & $0.13(1)$ & $0.00(1)$ \\
\hline Less than once a week or never & $548(90.3)$ & $7681(87.2)$ & & 1.00 & 1.00 \\
\hline More than once a week & $59(9.7)$ & $1131(12.8)$ & & $1.07(0.74-1.54)$ & $1.00(0.67-1.50)$ \\
\hline Regular exercise pattern & & & $20.92(1)^{* *}$ & $18.66(1)^{* *}$ & $10.58(1)^{* *}$ \\
\hline Yes & $394(65.1)$ & $6465(73.7)$ & & 1.00 & 1.00 \\
\hline No exercise at all & $211(34.9)$ & $2313(26.3)$ & & $1.62(1.30-2.02)$ & $1.51(1.18-1.93)$ \\
\hline Seen a doctor in past 4 weeks & & & $65.72(1)^{* *}$ & $35.67(1)^{* *}$ & \\
\hline No & $196(31.0)$ & $4302(47.7)$ & & 1.00 & 1.00 \\
\hline More than once & $436(69.0)$ & $4726(52.3)$ & & $1.96(1.57-2.44)$ & $1.43(1.12-1.83)$ \\
\hline Seen a TCM practitioner in past 4 weeks & & & $14.86(1)^{* *}$ & $2.04(1)$ & $0.17(1)$ \\
\hline No & $469(74.2)$ & $7275(80.5)$ & & 1.00 & 1.00 \\
\hline More than once & $163(25.8)$ & $1758(19.5)$ & & $1.20(0.93-1.54)$ & $0.94(0.70-1.26)$ \\
\hline
\end{tabular}


Table 4 Associations between patient factors and doctor detection (Continued)

\begin{tabular}{|c|c|c|c|c|c|}
\hline Disease co-morbidity & & & $65.21(2)^{* *}$ & $17.64(2)^{* *}$ & $0.97(2)$ \\
\hline None & $221(36.4)$ & $4413(50.1)$ & & 1.00 & 1.00 \\
\hline One & $150(24.7)$ & $2216(25.1)$ & & $1.29(0.97-1.72)$ & $1.05(0.76-1.44)$ \\
\hline Two or more & $236(38.9)$ & $2188(24.8)$ & & $1.88(1.40-2.53)$ & $1.18(0.84-1.65)$ \\
\hline Family history mental illness & & & $70.56(1)^{* *}$ & $48.24(1)^{* *}$ & $9.53(1)^{* *}$ \\
\hline No & $483(81.5)$ & $7919(91.7)$ & & 1.00 & 1.00 \\
\hline Yes & $110(18.5)$ & $721(8.3)$ & & $2.61(1.99-3.43)$ & $2.61(1.99-3.43)$ \\
\hline Service sector & & & $2042(1)$ & $1.89(1)$ & $4.34(1)^{*}$ \\
\hline Public & $183(28.8)$ & $2355(26.0)$ & & 1.00 & 1.00 \\
\hline Private & $453(71.2)$ & $6714(74.0)$ & & $1.20(0.93-1.55)$ & $1.36(1.02-1.83)$ \\
\hline Symptom severity/PHQ-9 score ${ }^{c}$ & & & $779.72(4)^{* *}$ & & $147.03(4)^{* *}$ \\
\hline Minimal/score 0-4 & $175(29.1)$ & $5725(65.0)$ & & & 1.00 \\
\hline Mild/score 5-9 & $178(29.6)$ & $2260(25.6)$ & & & $2.16(1.63-2.86)$ \\
\hline Moderate/score 10-14 & $135(22.4)$ & $622(7.1)$ & & & $4.67(3.34-6.53)$ \\
\hline Mod-severe/score 15-19 & $72(12.0)$ & $162(1.8)$ & & & $8.53(5.41-13.46)$ \\
\hline Severe/score 20-27 & $42(7.0)$ & $43(0.5)$ & & & $10.42(5.20-20.89)$ \\
\hline Self-reported depression historyc & & & $1564.17(1)^{* *}$ & & $85.05(1)^{* *}$ \\
\hline No & $314(52.8)$ & $8369(95.7)$ & & & 1.00 \\
\hline Yes & $281(42.6)$ & $378(4.3)$ & & & $10.16(7.73-13.34)$ \\
\hline Self-reported other mental illness history ${ }^{c}$ & & & $330.67(1)^{* *}$ & & $43.68(1)^{* *}$ \\
\hline No & $450(83.2)$ & $8425(97.6)$ & & & 1.00 \\
\hline Yes & $91(16.8)$ & $211(2.4)$ & & & $2.89(1.93-4.31)$ \\
\hline
\end{tabular}

The PCPs management patterns are outlined in Tables 5, 6 and 7.

\section{Discussion}

This is the first territory-wide study examining the diagnosis and management of depression amongst Hong Kong's primary care patients. A large scale survey such as this provides more accurate and actionable information when compared to surveys on selected clinical populations. Data on the rates of disease, barriers to care, identification of risk factors and service use is needed to help inform public policy and service planning [22]. This is one of the few studies performed in a predominantly Chinese population which includes both Chinese and non-Chinese subjects.

Although the prevalence of depressive disorders estimated in our study was similar to prevalence rates found in primary care settings in other parts of the world, by international standards, the detection rate of under one in every four patients who screened positive appears to be quite low [5]. Our study used a network of clinicians who had an active interest in mental health research. Of the 59 doctors who participated in the study, 14 had undertaken further diploma studies in community psychological medicine and all participants were aware that their patterns of diagnosis and management were being studied. Despite using this relatively biased sample of clinicians, detection rates were still lower than international standards suggesting that there may be factors aside from clinician skill which contribute to the low detection rates. It is well recognized that identification of depression in the primary care setting is challenging. Patients may not actively seek help or disclose any symptoms suggestive of a mood disorder, and the time available for the primary care consultation is often brief and mood disturbances may be missed [23]. PCPs often have difficulty in differentiating between psychological distress and psychiatric disorder due to difficulties in applying DSM-IV diagnostic criteria to primary care patients [24]. Relevant to our setting however is the further challenge of trying to identify depression in Chinese patients. The Chinese have been identified as an ethnic group with particularly low uptake rates of mental health services $[25,26]$. Chinese patients with depression often conceal their mood-related symptoms $[27,28]$ and may either deny having any depressive 
Table 5 Management of depression by doctors

\begin{tabular}{lcc}
\hline Depression management & $\mathbf{n ~ ( \% )}$ & PHQ-9 mean score (SD) \\
\hline $\begin{array}{l}\text { Patient currently under the } \\
\text { care of another professional }\end{array}$ & $189(30.6 \%)$ & $8.63(6.49)$ \\
Being observed & $172(27.8 \%)$ & $8.70(5.85)$ \\
Follow-up scheduled & $240(38.8 \%)$ & $9.22(6.11)$ \\
Medication prescribed & $313(50.6 \%)$ & $8.72(6.33)$ \\
Counselling provided & $259(41.9 \%)$ & $9.21(6.27)$ \\
Referral to health & $53(8.6 \%)$ & $12.08(6.45)$ \\
care professional &
\end{tabular}

Note. Management data was not available for another18 patients who were detected with depression. Percentage totals may exceed $100 \%$ as more than one management could be provided.

symptoms or may express them more somatically [28]. Many elderly Chinese patients perceive having a low mood to be a normal part of aging, and would not consider reporting depressive symptoms to their clinician [29,30]. Studies conducted in Europe and in America have noted lower detection rates for patients of Chinese descent than in their non-Chinese counterparts [25,31]. This finding appears to be confirmed in our study. When controlling purely for demographic factors, non-Chinese patients had a 2.5-fold increased likelihood of being diagnosed with depression. Even when controlling for severity and patientreported past history of depression and other mental illness, non-Chinese patients are still twice as likely to receive a diagnosis of depression by a doctor. In view of this finding, there may be a rationale to recommend screening

Table 6 Breakdown of subcategories of management provided

\begin{tabular}{lc}
\hline Breakdown of subcategory & $\mathbf{n}(\%)$ \\
\hline Medication prescribed (n=313) & $263(84.0 \%)$ \\
Antidepressant & $41(13.1 \%)$ \\
Z-class drug & $70(22.4 \%)$ \\
Benzodiazepine & $26(8.3 \%)$ \\
Anti-psychotic & \\
Counseling provided ( $\mathbf{n}=\mathbf{2 5 9 )}$ & $215(83.0 \%)$ \\
Supportive counseling & $49(18.9 \%)$ \\
Problem solving therapy & $31(12.0 \%)$ \\
Activity planning & $36(13.9 \%)$ \\
Cognitive behavioral therapy & \\
Referral to health care professional (n=53) & $4(7.5 \%)$ \\
Private psychiatrist & $7(13.2 \%)$ \\
Psychologist & $12(22.6 \%)$ \\
Government-funded psychiatric service & $18(34.0 \%)$ \\
Counsellor & $1(1.9 \%)$ \\
Emergency department & $3(5.7 \%)$ \\
Social worker &
\end{tabular}

Note. Percentage totals may exceed $100 \%$ due to multiple responses.
Table 7 Management of depression by doctors as classified by depression history

\begin{tabular}{lcc}
\hline & $\begin{array}{c}\text { With depression } \\
\text { history } \\
\mathbf{n = 2 7 4}\end{array}$ & $\begin{array}{c}\text { No depression } \\
\text { history } \\
\boldsymbol{n}=\mathbf{3 0 3}\end{array}$ \\
\hline $\begin{array}{l}\text { Depression management (n, \%) } \\
\begin{array}{l}\text { Patient currently under the } \\
\text { care of another professional }\end{array}\end{array}$ & $126(46.0 \%)$ & $54(17.8 \%)$ \\
$\begin{array}{l}\text { Being observed } \\
\text { Follow-up scheduled }\end{array}$ & $41(15.0 \%)$ & $120(39.6 \%)$ \\
$\begin{array}{l}\text { Medication prescribed } \\
\text { Counselling provided }\end{array}$ & $106(38.7 \%)$ & $117(38.6 \%)$ \\
$\begin{array}{l}\text { Referral to health care } \\
\text { professional }\end{array}$ & $110(40.1 \%)$ & $126(41.6 \%)$ \\
Mean PHQ-9 score (Mean \pm SD) & $9.39 \pm 6.35^{\dagger}$ & $129(42.6 \%)$ \\
\hline
\end{tabular}

Note. Percentage totals may exceed $100 \%$ as more than one management could be provided.

${ }^{\dagger}$ Independent t-test: $t=2.19, d f=527, p=0.029$.

of patients to help improve detection rates in Chinese patients.

Lack of continuity of care is another factor that can contribute to low detection rates. Studies have shown that many PCPs are reluctant to label a patient as depressed on a single consultation, but rather over a period of observation [32]. Many patients in Hong Kong do not have a regular doctor, opting for convenience and accessibility rather than continuity of care when seeking medical attention [13]. In a study on public sector primary care patients, only $10 \%$ reported to know their consulting doctor well [33]. In this study, patients who were most likely to receive a diagnosis of depression included those who were female, aged over 55 years, retired or housewives, and those who had seen a doctor within the last month. Patients with these characteristics are more likely to receive regular medical attention, making it easier for doctors to detect the presence of depressive symptoms [34].

In this study, it appears doctors in private practice may be more willing or able to diagnose depression. With symptom severity and patient-reported past history of depression controlled in the analysis, private sector patients were 1.36 times more likely to receive a depression diagnosis than those in the public sector. With an average consultation time of only 5.5 minutes [33], by international standards, primary care consultations in Hong Kong's public sector clinics are brief [35]. As the main focus of these services is usually on chronic disease care, there may be insufficient time to explore psycho-social issues [13]. A recent meta-analysis found that consultations associated with a diagnosis of a psychological problem tended to be longer than those without any psychological diagnosis [36].

Our examination of doctors' management patterns found over $50 \%$ of patients diagnosed with depression were 
prescribed medication. In Hong Kong, drugs are dispensed by the doctor rather than through a pharmacy which, in association with a lack of access to psychosocial services, are likely to be key reasons for the high rates of prescribing. Another reason for the high rates of drug prescribing may also be the doctor's perception of patient expectations for medication. In a telephone survey of Hong Kong Chinese community members, although only $40 \%$ of respondents believed they always needed drugs to treat an illness, $76 \%$ expected to receive a prescription every time they saw a doctor. In almost $100 \%$ of cases, during their most recent doctor visit, at least one medication had been prescribed. The study concluded that doctors in Hong Kong over-estimate patients' expectation for medications, and their prescribing habits may have subsequently produced a high expectation for medications by patients [37]. A more focused study is needed to better examine doctor's prescribing practices and their perception of what constitutes best practice for managing primary care patients with depression.

Examination of the doctor's referral patterns found that $8.6 \%$ of patients diagnosed with depression were referred for other services with $34 \%$ of new referrals to counselors, $23 \%$ to public hospital clinics and only $7.5 \%$ to private psychiatrists. As in many other settings, access to psychosocial services in Hong Kong is very limited. In addition, Hong Kong also has very few specialist psychiatrists. In 2005 the population to specialist ratio for psychiatrists in Hong Kong was 1:44,202, far higher than the UK where the ratio was 1: 16,836 [38]. Of these, only a small proportion of psychiatrists practice in the private sector [12]. In Hong Kong, patients can directly consult specialists in the private sector without a primary care referral. When asked who they would seek help from if they thought they were depressed, it has been reported that $20 \%$ of patients in Hong Kong would prefer to see a psychiatrist [39]. This has significant service implications as patients may potentially 'by-pass' seeing a primary care doctor, leading to excessive demands for already stretched specialist services.

\section{Strengths and limitations}

One of the major strengths of this study was our success in recruiting a large number of primary care physicians to collaborate in this study. There are many service delivery options for patients seeking primary care in Hong Kong and our wide sampling of practice types captures this diversity. Despite this, there were limitations to our sampling strategy. As there is no comprehensive registry of primary care providers for Hong Kong, the mailing list of the Hong Kong College of Family Physicians (HKCFP) was chosen as the primary sample frame for the doctors. A limitation is that doctors who provide primary care, but are not members of the HKCFP were not deliberately sampled. The doctors who participated in this study were volunteers who joined our research network, and there was a biased sampling towards doctors with an interest in mental health. The results of this study are likely to reflect a "best case scenario" with better detection rates and more optimal treatment than is being offered in Hong Kong's wider primary care setting. The low referral rates found in this study may have been a result of this sampling bias as a high proportion of the doctors had postgraduate training in psychological medicine and may have felt more capable of providing depression care than doctors without such training.

Whilst we recruited subjects from all three regions of Hong Kong, approximately 40\% were recruited from Hong Kong Island, the smallest in terms of population size. Although the three regions differ in terms of geographic size, population number and socio-economy, a previous study of Hong Kong's primary care found the morbidity patterns to be quite similar territory wide [40].

Although we were able to obtain a response rate of over $80 \%$, no clinical information was available for nonrespondents due to the lack of electronic medical records in most primary care clinics and ethical constraints on patient data privacy. Of the non-respondents $62.7 \%$ were female (vs $56.5 \%$ in respondents); $83.5 \%$ refused for no reason, $10.1 \%$ cited that they felt too sick, $6.1 \%$ cited that they did not have enough time, and $0.3 \%$ refused due to hearing difficulties.

Screening for depression was based on a subjective self-reported instrument and was not confirmed by a clinical diagnostic interview which would be the gold standard for diagnosis of depression.

\section{Conclusions}

Around one in ten Hong Kong primary care patients screen positive for depression, in which doctors diagnose depression in approximately one in four. Patients with a past history of depression, who are unemployed, or who have multiple co-existing illnesses appear to be at greatest risk of being depressed. Patients with a past history of depression or who have severe symptoms of depression are more likely to receive a diagnosis of depression. Chinese patients are half as likely to receive a diagnosis of depression by a doctor as non-Chinese patients. In patients diagnosed with depression, over half are managed with medication. The most commonly used referral service by primary care doctors are to counselors. Further studies are needed to examine doctors prescribing practices and their perceptions of what constitutes best practice for managing primary care patients with depression.

\section{Competing interests}

The authors declare that they have no competing interests. 


\section{Authors' contributions}

$\mathrm{CL}$ initially conceived the study. All authors collectively designed and drafted the study protocol and sought funding and ethical approving. DF led on statistical analyses. CL, TPL, YL, BC, SW contributed to recruitment and data collection. KC was the project coordinator, recruited and trained the fieldworkers, assisted with recruitment of study doctors, coordinated the data collection, and contributed to the drafting of the manuscript. WYC is $\mathrm{PI}$ of the funding application, drafted the study protocol, coordinated the research network and research team, and drafted the manuscript. All authors have read the draft critically and approved the final manuscript.

\section{Acknowledgements}

This study was funded by the Food and Health Bureau Commissioned Research on Mental Health Policy and Service Ref No. SMH-27.

The authors wish to acknowledge the contributions of Professor Jane Gunn (University of Melbourne) and Professor Walter Rosser (Queens University, Ontario) who helped as external advisors during the planning and drafting stages of the original study protocol. We also wish to thank all the doctors of our Primary Care Research Network for their continuing collaboration with us on this project.

Much appreciation goes to the many research assistants who assisted in this project, including Horace Cheung, Emily Chan, Joanne Kong, Vivian Chau, Gladys Ng, Chloe Ling, Amy Li, Henrietta Lai, Winnie Tan, Meryl Lam, Carrie Li, and student helpers in particular Marie Kam and Apple Chui.

\section{Author details}

${ }^{1}$ Department of Family Medicine and Primary Care, The University of Hong Kong, Hong Kong, China. ${ }^{2}$ Division of Family Medicine and Primary Health Care, School of Public Health and Primary Care, The Chinese University of Hong Kong, Hong Kong, China. ${ }^{3}$ School of Nursing, The University of Hong Kong, Hong Kong, China. ${ }^{4}$ Department of Family Medicine, Hong Kong West Cluster, Hospital Authority, Hong Kong, China. ${ }^{5}$ Family Medicine and Primary Care Centre, Hong Kong Sanatorium and Hospital, Hong Kong, China.

Received: 15 July 2013 Accepted: 3 February 2014

Published: 12 February 2014

\section{References}

1. Murray CJ, Lopez AD: The global burden of disease: a comprehensive assessment of morbidity and disability from disease, injuries and risk factors in 1990 and projected to 2020. Cambridge, MA: Harvard University Press; 1996.

2. Culpepper L: The active management of depression. J Fam Pract 2002, 51(9):769-776.

3. Nutting PA, Rost K, Dickinson M, Werner JJ, Dickinson P, Smith JL, Gallovic B: Barriers to initiating depression treatment in primary care practice. J Gen Intern Med 2002, 17(2):103-111.

4. Nutting PA, Rost K, Smith J, Werner JJ, Elliot C: Competing demands from physical problems: effect on initiating and completing depression care over 6 months. Arch Fam Med 2000, 9(10):1059-1064.

5. Mitchell AJ, Vaze A, Rao S: Clinical diagnosis of depression in primary care: a meta-analysis. Lancet 2009, 374(9690):609-619.

6. Yu X, Tam WWS, Wong PTK, Lam TH, Stewart SM: The patient health questionnaire-9 for measuring depressive symptoms among the general population in Hong Kong. Compr Psychiatry 2012, 53:95-102.

7. Population Health Survey 2003/2004. Collaborative Project of Department of Health and Department of Community Medicine, University of Hong Kong. Available at http://www.chp.gov.hk/files/pdf/report_on_population_ health_survey_2003_2004_en.pdf.

8. Middleton H, Shaw I, Feder G: NICE guidelines for the management of depression. Br Med J 2005, 330:267-268.

9. Mitchell J, Trangle M, Degnan B, Gabert T, Haight B, Kessler D, Mack N, Mallen E, Novak H, Rossmiller D, Setterlund L, Somers K, Valentino N, Vincent S: Institute for clinical systems improvement. In Adult Depression in Primary Care. Updated September 2013. Available at https://www.icsi.org/_asset/ fnhdm3/Depr-Interactive0512b.pdf.

10. New Zealand Guidelines Group: Identification of common mental disorders and management of depression in primary care. An evidence-based best practice guideline. Wellington: New Zealand Guidelines Group; 2008.

11. Mathews G, Lu D, Ma J: Hong Kong, China: learning to belong to a nation. In 2008. London and New York: Routledge; 2008.
12. Cheung EFC, Lam LCW, Hung SF: Hong Kong. In International Perspectives on Mental Health. Edited by Ghodse H. London: RCPsych Publications; 2011.

13. Wun YT, Lam TP, Lam KF, Goldberg D, Li DKT, Yip KC: How do patients choose their doctors for primary care in a free market? J Eval Clin Pract 2010, 16(6):1215-1220.

14. Chin W, Lam C, Wong S, Lo Y, Fong D, Lam T, Lee P, Wong J, Chiu B, Chan K: The epidemiology and natural history of depressive disorders in Hong Kong's primary care. BMC Fam Pract 2012, 12(1):129.

15. Kroenke K, Spitzer RL, Williams JB: The PHQ-9: validity of a brief depression severity measure. J Gen Intern Med 2001, 16(9):606-613.

16. Cheng $C M$, Cheng $M$ : To validate the Chinese version of the $2 Q$ and PHQ-9 questionnaires in Hong Kong Chinese patients. Hong Kong Pract 2007, 29:381-390.

17. Zoberi K, Niemieic RM, Margolic RB: Teaching integrated behavioural health in primary care clerkship. Med Teach 2008, 30:e218-e223.

18. Lam CLK, Leung GM, Mercer SW, Fong DYT, Lee A, Lam TP, Lo YYC: Utilization pattern of primary care services in Hong Kong - does having a family doctor make a difference. Hong Kong Med J 2011, 17(3):S28-S32.

19. Gunn JM, Gilchrist GP, Chondros P, Ramp M, Hegarty KL, Blashki GA, Pond DC, Krios M, Herrman HE: Who is identified when screening for depression is undertaken in general practice? baseline findings from the diagnosis, management and outcomes of depression in primary care (diamond) longitudinal study. MJA 2008, 188(12):s119-s125.

20. Tabachnick BG, Fidell LS: Using multivariate statistics. 6th edition. Boston: Allyn and Bacon; 2013.

21. Food and Health Bureau HKS: My health My choice: health reform second stage consultation document. Hong Kong: Government Logistics Department; 2010

22. Jenkins R: Making psychiatric epidemiology useful: the contribution of epidemiology to government policy. Acta Psychiatr Scand 2001, 103(1):2-14

23. Rubenstein LV, Jackson-Triche $M$, Unutzer J, Miranda J, Minnium K, Pearson $\mathrm{ML}$, Wells KB: Evidence-based care for depression in managed primary care practices. Health Aff (Millwood) 1999, 18(5):89-105.

24. van Rijswijk E, van Hout $H$, van de Lisdonk E, Zitman F, van Weel C: Barriers in recognising, diagnosing and managing depressive and anxiety disorders as experienced by family physicians; a focus group study. BMC Fam Pract 2009, 10(1):52

25. Leong FL, Lau AL: Barriers to providing effective mental health services to Asian Americans. Ment Health Serv Res 2001, 3(4):201-214.

26. Wong DFK, Tsui HKP, Pearson V, Chen EYH, Chiu SN: Family burdens, Chinese health beliefs, and the mental health of Chinese caregivers in Hong Kong. Transcult Psychiatry 2004, 41(4):497-513.

27. Stewart D, Gucciardi E, Grace S: Depression. BMC Womens Health 2004, 4(Suppl 1):S19.

28. Parker G, Gladstone G, Chee KT: Depression in the planet's largest ethnic group: the Chinese. Am J Psychiatry 2001, 158:857-864.

29. Chen R, Copeland JRM, Wei L: A meta-analysis of epidemiological studies in depression of older people in the People's Republic of China. Int J Geriatr Psychiatry 1999, 14(10):821-830

30. Vasiliadis H-M, Lesage A, Adair C, Wang PS, Kessler RC: Do Canada and the United States differ in prevalence of depression and utilization of services? Psychiatr Serv 2007, 58(1):63-71.

31. Liu CH, Ingleby D, Meeuwesen L: Barriers to health care for Chinese in the Netherlands. Int J Fam Med 2011, 2011:635853.

32. Kessler D, Bennewith O, Lewis G, Sharp D: Detection of depression and anxiety in primary care: a follow up study. BMJ 2002, 325:1016-1017.

33. Mercer S, Fung C, Chan F, Wong F, Wong S, Murphy D: The Chineseversion of the CARE Measure reliably differentiates between doctors in primary care: a cross-sectional study in Hong Kong. BMC Fam Pract 2011, 12(1):43

34. Bushnell J, Ma GRG: Frequency of consultations and general practitioner recognition of psychological symptoms. Br J Gen Pract 2004, 54(508):838-843.

35. Deveugele M, Derese A, Brink-Muinen A, Bensing J, Maeseneer JD: Consultation length in general practice: cross sectional study in six European countries. BMJ 2002, 325(7362):472.

36. Hutton C, Gunn J: Do longer consultations improve the management of psychological problems in general practice? A systematic literature review. BMC Health Serv Res 2007, 7(1):71.

37. Lam CL, Catarivas MG, Lauder IJ: A pill for every ill. Fam Pract 1995 12(2):171-175

38. Hong Kong College of Psychiatrists: Submission of the Hong Kong College of Psychiatrists to the Panel on Health Services of the Legislative Council on 
Mental Health Policy in Hong Kong. Legislative Council paper Paper No. CB (2) 373/07-08(05). Hong Kong: Hong Kong College of Psychiatrists; 2007.

39. Wun YT, Lam TP, Goldberg D, Lam KF, Li KT, Yip KC: Reasons for preferring a primary care physician for care if depressed. Fam Med 2011, 43(5):344-350.

40. Lo YY, Lam CL, Mercer SW, Fong DY, Lee A, Lam TP, Lee R, Chiu B, Tang J, Chui $B$, et al: Patient morbidity and management patterns of communitybased primary health care services in Hong Kong. Hong Kong Med J 2011, 17(3 Suppl 3):33-37.

doi:10.1186/1471-2296-15-30

Cite this article as: Chin et al:: Detection and management of depression in adult primary care patients in Hong Kong: a cross-sectional survey conducted by a primary care practice-based research network. BMC Family Practice 2014 15:30.

\section{Submit your next manuscript to BioMed Central and take full advantage of:}

- Convenient online submission

- Thorough peer review

- No space constraints or color figure charges

- Immediate publication on acceptance

- Inclusion in PubMed, CAS, Scopus and Google Scholar

- Research which is freely available for redistribution 